\title{
A New Synthetic Diet Increased Bone Mineral Density and Strength of Ovariectomized Rat
}

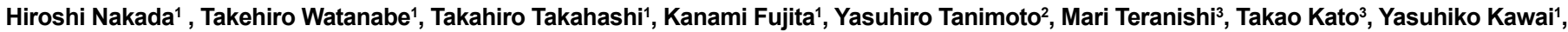
Toshiro Sakae ${ }^{4}$ and Racquel Z LeGeros ${ }^{5}$

${ }^{1}$ Department of Removable Prosthodontics, Nihon University School of Dentistry at Matsudo, Chiba 271-8587, Japan

${ }^{2}$ Department of Dental Biomaterials, Nihon University School of Dentistry at Matsudo, Chiba 271-8587, Japan

${ }^{3}$ Department of Oral Implantology, Nihon University School of Dentistry at Matsudo, Chiba 271-8587, Japan

${ }^{4}$ Department of Histology, Nihon University School of Dentistry at Matsudo, Chiba 271-8587, Japan

${ }^{5}$ Department of Biomaterials and Biomimetics, New York University College of Dentistry, New York 10010, USA

\begin{abstract}
This study aimed to determine changes in Bone Mineral Density (BMD) and bone strength of ovariectomized rats fed a synthetic diet of $\mathrm{Mg}, \mathrm{Zn}$, and $\mathrm{F}$ incorporated with carbonate apatite. Group $1(n=4)$ was fed a Normal Mineral Diet (NMD) and Group 2 ( $n=4$ ) was fed the Synthetic Diet (SD: 0.74\% Ca, 0.48\% P, 0.10\% Mg, 365.4 ppm Zn, $51.01 \mathrm{ppm}$ $\mathrm{F}$ and $0.27 \% \mathrm{CO}_{3}$ ). Rats were sacrificed after eight weeks and the femur was isolated. The micro-CT results showed that values of BMD for Group 2 were higher than those of Group 1. Bone strength values of Group 2 measured by a 3 -point bending test were significantly higher than those of Group 1. These results indicated that Group 2 stimulated bone quality, increased BMD and bone strength. The SD has the potential to prevent bone loss and as an osteoporosis therapy.
\end{abstract}

Keywords: Synthetic carbonate apatite diet; Ovariectomized rat; Bone mineral density; Bone strength; Micro-CT

\section{Introduction}

Osteoporosis is a systemic disease that results in reduced bone mass and poor trabecular structure. It is defined as "a skeletal disorder characterized by compromised bone strength predisposing to an increased risk of fracture [1]". Bone strength is associated with 2 factors: bone density and bone quality, contributing about $70 \%$ and $30 \%$ respectively. Bone tissue quality is related to the microstructure, metabolic turnover, micro-damage, calcification, and collagen crosslinking. Bone density is an important parameter in predicting fracture. However, fracture risk increases with age, even if bone density remains constant [2]. Therefore, it is necessary to improve not only bone density but also bone quality, including bone strength.

LeGeros' research group developed a calcium phosphate-based supplement (synthetic diet: SD) for osteoporosis treatment in recent years [3]. The SD consists of magnesium $(\mathrm{Mg})$, zinc $(\mathrm{Zn})$ and fluoride (F) ions incorporated in the carbonate apatite.

This study aimed to determine changes in Bone Mineral Density (BMD) and strength induced by the SD using micro-CT and the 3-point bending test.

\section{Materials and Methods}

\section{Experimental animals}

Eight female Wistar rats (Sankyo Labo Service Co., Tokyo, Japan) 19 weeks-old, were used. The experimental protocol was approved by the animal experiment ethics committee of the School of Dentistry at Matsudo. The eight rats were randomly distributed into 2 groups $(n=4$ per group). Group 1consisted of ovariectomized rats (OVX) provided with a normal mineral diet (NMD, Table 1, AIN-93M, Nosan Co., Yokohama, Japan). Group 2 consisted of OVX rats provided with the SD (Table 1, Oriental Yeast Co., Tokyo, Japan). The body weights of all rats were measured at baseline and 8 weeks after surgery. All animals were then euthanized with carbon dioxide at 8 weeks after surgery, and bilateral femora were removed.
Measurements of bone mineral density (BMD) and threedimensional color mapping (3D-map)

Micro-CT settings: Femoral micro-CT images (Figure 1A) were acquired setting the measurement range at the middle point region of the full femoral length measured using calipers (Figure 1B). The microCT was carried out using a R_mCT (Rigaku Co., Japan). The value of BMD was measured using TRI/3D-Bon BMD software (TRI/3D; Ratoc System Engineering Co., Japan). Three-dimensional color mapping (3D-map) of the BMD distributions was carried out using TRI/3D image analysis software.

\section{Measurements of bone strength}

Bone strength was measured using an Instron type testing machine system (Figure 1C), TG-5kN, Minebea Co., Japan). Bending force was loaded on the middle point of the bone length at $5.0 \mathrm{~mm} / \mathrm{min}$.

\section{Statistical analysis}

The means and standard deviations of body weight, BMD, and bone strength were calculated for each group and compared using Student's t-test.

\begin{tabular}{|l|c|c|}
\hline Group 1 & 20 Weeks Old (After surgery) & 28 Weeks old (After 8 weeks) g \\
\hline Group 2 & $181.9 \pm 1.9$ & $239.8 \pm 13.6$ \\
\hline & $182.7 \pm 3.2$ & $233.6 \pm 12.9$ \\
\hline
\end{tabular}

Table 1: Body weight (g) gain for the 2 groups after the surgery and 8 weeks.

*Corresponding author: Hiroshi Nakada, Department of Removable Prosthodontics, Nihon University School of Dentistry at Matsudo, Chiba 271-8587, Japan, E-mail: nakada.hiroshi@nihon-u.ac.jp

Received June 08, 2013; Accepted July 22, 2013; Published August 22, 2013

Citation: Nakada H, Watanabe T, Takahashi T, Fujita K, Tanimoto Y, et al. (2013) A New Synthetic Diet Increased Bone Mineral Density and Strength of Ovariectomized Rat. Bioceram Dev Appl S1: 003. doi: 10.4172/22090-5025.S1-003

Copyright: (C) 2013 LNakada H, et al. This is an open-access article distributed under the terms of the Creative Commons Attribution License, which permits unrestricted use, distribution, and reproduction in any medium, provided the original author and source are credited. 
Citation: Nakada H, Watanabe T, Takahashi T, Fujita K, Tanimoto Y, et al. (2013) A New Synthetic Diet Increased Bone Mineral Density and Strength of Ovariectomized Rat. Bioceram Dev Appl S1: 003. doi: 10.4172/22090-5025.S1-003

Page 2 of 3

\section{Results}

\section{Body weight}

Table 1 shows body weight measurements for the 2 groups at each observation time. The weights subsequently increased until after 8 weeks.

\section{BMD}

Changes in BMD with time for each group are shown in Table 2. The BMD values for the 2 groups at 8 weeks were as follows: NMD: $1215.8 \pm 6.4 \mathrm{mg} / \mathrm{cm}^{3}$ and SD: $1218.9 \pm 10.1 \mathrm{mg} / \mathrm{cm}^{3}$. The BMD was not significantly for 2 groups at 8 weeks. But Group 2 became a trend higher than BMD.

\section{Bone strength}

Changes in Bone strength with time for each group are shown in Table 2. The bone strength values for the 2 groups at 8 weeks were as follows: Group 1: $148.5 \pm 7.7 \mathrm{~N}$ and Group 2:165.7 $\pm 5.3 \mathrm{~N}$. The bone strength was significantly higher for Group 2compared with Group1.

\begin{tabular}{|l|c|c|}
\hline & BMD $\left(\mathbf{m g} / \mathbf{c m}^{3}\right)$ & Bone Strength (N) \\
\hline Group 1 & $1215.8 \pm 6.4$ & $148.5 \pm 7.7$ \\
\hline Group 2 & $1218.9 \pm 10.1$ & $165.7 \pm 5.3$ \\
\hline
\end{tabular}

Table 2: Bone mineral density and bone strength of femoral mid-shaft for the 2 groups 8 weeks after the surgery.

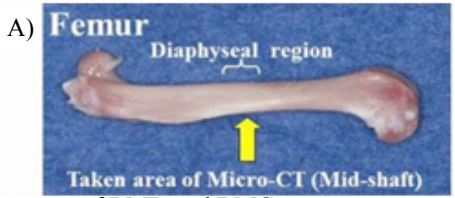

$\begin{array}{ll}\text { B) Measurement area of BMD and BMC } & \text { C) Measurement of bone strength }\end{array}$
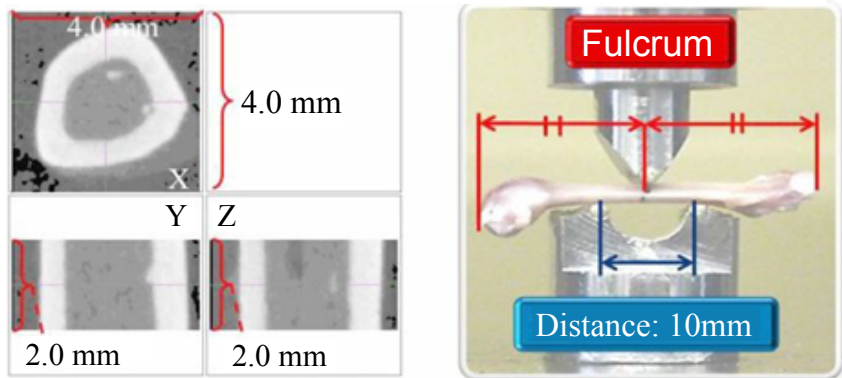

Figure 1: A) Image of Femur, B) Measurement area using Micro-CTin femur and C) Image of measurement of bone strength.

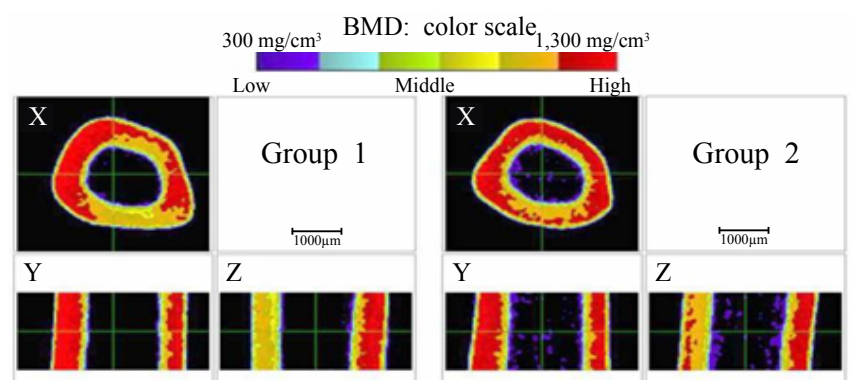

Figure 2: 3D-maps of the femoral mid-shift region for the 2 groups 8 weeks after surgery. Red and orange indicate high bone mineral density, yellow and green indicate medium bone mineral density, and light blue and purple indicate low bone mineral density.

\section{D-map}

Figure 2 shows the 3D-map for the 2 groups after 8 weeks. For Group 1, the density of the exterior of the cortical bone was high, and the density of the region adjacent to the marrow cavity was low. The values of Group 2 were similar. For Z-axis, density of Group 2 indicated higher than Group 1.

\section{Discussion}

LeGeros R.Z. had carried out many researches on calcium phosphate-based bone substitute materials that are used for teeth or bone replacement, bone repair, augmentation, or regeneration [46]. The present study using rats demonstrates the effects of estrogen deficiency (ovariectomy) alone or combined with SD on bone quality (density and strength) as assessed by 3D-mapand the 3-point bending test. The analytical system employing micro-CT and TRI/3D used in this study is capable of $3 \mathrm{D}$ visualization of the bone structure, qualitative analysis by pseudocolor rendering, and quantitative analysis of BMD [7-9]. In conventional micro-CT imaging, black and white images are evaluated because the color intensity representing X-ray penetration and non-penetration through materials is judged. Moreover, it is difficult to observe changes in bone quality with regard to the BMD presented in 3D-map in conventional X-ray or pathological images, suggesting the usefulness of this analytical system including microCT. Furthermore, much useful information can be obtained by a single determination because this analytical system is capable of qualitative and quantitative analyses.

Using SD, Mijares et al. [3] reported that rats received SD had shown more bone formation and calcification of the mandible bone compared with the rats received NMD. The results of our present study support these findings. This experiment was able to the relationship between the calcification and the bone change. More importantly this study demonstrated the therapeutic efficacy of SD preparations when used as a supplement to a NMD. Ingested SD may have the potential as an osteoporosis therapeutic agent induced by estrogen deficiency.

\section{Conclusion}

The extra-elements incorporated carbonate apatite diet successfully recovered the bone mineral density and strength for the ovariectomized rats. The results indicated that the new synthetic diet may be one of the selections for osteoporotic therapeutic agent.

\section{Acknowledgment}

This study was supported in part by Grants-in-Aid for Scientific Young Scientists (B) (23792297, M. T.) from MEXT, Japan. This research was started in collaboration with Prof. Racquel Z. LeGeros.

\section{References}

1. NIH Consensus Development Panel on Osteoporosis Prevention, Diagnosis, and Therapy (2001) Osteoporosis prevention, diagnosis, and therapy. JAMA 285: 785-795.

2. Leslie WD, Morin S, Lix LM, Johansson H, Oden A et al. (2012) Fracture risk assessment without bone density measurement in routine clinical practice. Osteoporos Int 23: 75-85.

3. Mijares D, Kulkarni A, Lewis K, Yao F, Xi Q, et al. (2012) Oral bone loss induced by mineral deficiency in a rat model: effect of a synthetic bone mineral (SBM) preparation. Arch Oral Biol 57: 1264-1273.

4. Daculsi G, Bouler JM, LeGeros RZ (1997) Adaptive crystal formation in normal and pathological calcifications in synthetic calcium phosphate and related biomaterials. Int Rev Cytol 172: 129-191.

5. LeGeros RZ (2008) Calcium phosphate-based osteoinductive materials. Chem Rev 108: 4742-4753. 
Citation: Nakada H, Watanabe T, Takahashi T, Fujita K, Tanimoto Y, et al. (2013) A New Synthetic Diet Increased Bone Mineral Density and Strength of Ovariectomized Rat. Bioceram Dev Appl S1: 003. doi: 10.4172/22090-5025.S1-003

6. Tokudome Y, Otsuka M, Ito A, LeGeros RZ (2009) Long-term therapeutic effect of novel calcium phosphate-based compounds injected in ovariectomized rats. J Biomed Mater Res B Appl Biomater 90: 229-237.

7. Nakada H, Suzuki S, Sakae T, Tanimoto Y, Kuboyama N, et al. (2011) Quantitative and qualitative analyses of low-mineral-diet ovariectomised rat femora using microscopic computed tomography. J Hard Tissue Biol 20: 107-114.
8. Suzuki S, Nakada H, Sakae T, Tanimoto Y, Kawai Y, et al. (2012) Bone quality of the femoral mid-shaft of ovariectomized rats fed a low-mineral diet. J Hard Tissue Biol 21: 245-256.

9. Sakae T, Nakada H, Teranishi M, Kato T, Suzuki S, et al. (2012) Comparison between the lateral and medial femur in low-mineral-diet-fed ovariectomized rats using Raman spectral analysis. Key Eng Mater 529-530. 337-340. 